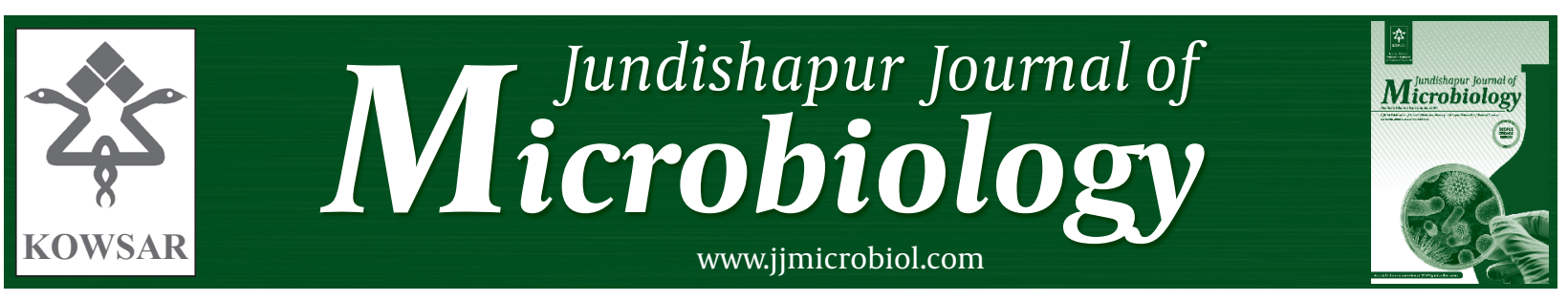

\title{
Comparing the Frequency of Mycobacterium Tuberculosis With Direct Microscopy and Culture Methods
}

\author{
Reza Akhavan ${ }^{1}$, Zahra Meshkat ${ }^{2 *}$, Saeed Amel Jamehdar ${ }^{3}$ \\ ${ }^{1}$ School of Medicine, Mashhad University of Medical Sciences, Mashhad, IR Iran \\ ${ }^{2}$ Women's Health Research Center, Mashhad University of Medical Sciences, Mashhad, IR Iran \\ ${ }^{3}$ Microbiology and Virology Research Center, Mashhad University of Medical Sciences, Mashhad, IR Iran
}

\section{A R T I C L E I N F O}

Article type:

Letter to Editor

Article history:

Received: 09 Mar 2012

Revised: 11 Apr 2012

Accepted:14 Apr 2012

Keywords:

Mycobacterium tuberculosis

Tuberculosis Diagnosis

Sensitivity and Specificity

\section{Dear Editor,}

Tuberculosis has been a threat for the human's life all over the history. While other epidemics last only weeks to moths, tuberculosis (TB) epidemic has been continuing for centuries.

Twenty two countries were announced as the countries with the highest incident rate including $80 \%$ of new cases. Our country, Iran, is not among theses 22 (1); however, tuberculosis is one of the greatest health problems in Iran. Although the principles of treatment had been known since 50 years and short course treatment is practiced for 20 years, yet many patients are not diagnosed and don't receive proper treatment in many regions of the country (2).

Although many countries have conducted several studies about tuberculosis prevalence, there are few such studies done in our country. For a good estimation of the disease burden we first have to diagnose it correctly. According to
- Please cite this paper as:

Akhavan R, Meshkat Z, Amel Jamehdar S. Comparing the Frequency of Mycobacterium Tuberculosis With Direct Microscopy and Culture Methods. Jundishapur J Microbiol. 2013;6(1):95-6. DOI: $10.5812 / \mathrm{jjm} .4596$

Published by Kowsar Corp, 2013. cc 3.0.

the shortage of resources in our country, the most diagnosis measures practiced in the area are direct microscopy for acid fast bacilli (AFB). In this study, we evaluated the prevalence of smear positive samples and compared the results with the results obtained from culture method.

Our data showed the overall sensitivity and false negative results of microscopic examination were $66.0 \%$ and $34.0 \%$, respectively. In addition, the overall specificity and false positive results would be $94.6 \%$ and $5.4 \%$, respectively. The measure of agreement between obtaining results by microscopy and culture methods was 0.484. There was a report for comparing the positive samples in microscopy and culture methods regarding the culture as a gold standard method and AFB were found in only $46 \%$ of culture positive smears that is lower than our results (3). In other studies AFB were found in the range of 53\%-80\% of the first smear of positive sputum samples (4). In Down study, the sensitivity and specificity of the sputum smears compared to the culture

\footnotetext{
${ }^{*}$ Corresponding author: Zahra Meshkat, Women's Health Research Center, Mashhad University of Medical Sciences, Mashhad, IR Iran. Tel: +98-5118012453, Fax: +98-5118002960, E-mail: meshkatz@mums.ac.ir permits unrestricted use, distribution, and reproduction in any medium, provided the original work is properly cited.
} 
method were $80 \%$ and $94.1 \%$, respectively, which is in agreement with our results (5). Our data renders the microscopic method as a non-expensive and suitable method for diagnosis of tuberculosis.

\section{Acknowledgments}

This study was a thesis presented for the degree of Medical Doctor (MD) which supported by Mashhad University of Medical Sciences, Mashhad, Iran (grant No. 87749 and thesis No. 6383).

\section{Financial Disclosure}

The authors report no conflicts of interest. The authors are responsible for the content of the paper.

\section{Funding/Support}

This work was supported by a grant (No: 87571) of Mash- had University of Medical Sciences, Mashhad, IR Iran.

\section{Authors' Contribution}

None declared.

\section{References}

1. World Health Organization. Global tuberculosis control: a short update to the 2009 report. 2009. World health organization: geneva. 2010.

2. Amani Firouz BJ, Sabzevari A, Garousi B, Nahan Moghadam N. Epidemiology of tuberculosis in ardabil, 2001-2005. JAUMS. 2007.

3. Nelson SM, Deike MA, Cartwright CP. Value of examining multiple sputum specimens in the diagnosis of pulmonary tuberculosis. JClin Microbiol. 1998;36(2):467-9.

4. Saleem S, Shabbir I, Iqbal R, Ullah Khan S. Value of three sputum smears microscopy in diagnosis of pulmonary tuberculosis. Pak JMed Res. 2007;46(4):1-5.

5. Down JA, O'Connell MA, Dey MS, Walters AH, Howard DR, Little MC, et al. Detection of Mycobacterium tuberculosis in respiratory specimens by strand displacement amplification of DNA. $J$ Clin Microbiol. 1996;34(4):860-5. 